\title{
Influence of dust parameters on Class II methanol maser pumping
}

\author{
Andrei B. Ostrovskii and Andrej M. Sobolev \\ Ural State University, Lenin str. 51, 620083 Ekaterinburg, Russia
}

\begin{abstract}
Results of model calculations for class II methanol masers (MMII) are presented. The model of the pumping assumes that an external dust layer provides the source of energy for maser excitation. The dependence of the emergent maser spectrum on the properties of the dust layer is studied. These properties include the chemical composition and sizes of dust grains, the temperature and optical depth of the dust layer. as well as the dilution factor of external dust emission. It is shown that, in order to reproduce the observed patterns of class II methanol maser line ratios, the pumping dust layer should be mostly composed of silicate grains with sizes smaller than $0.01 \mu$. It is shown that the layer of warm ( $>125 \mathrm{~K}$ ) dust effectively pumps the strongest MMII transitions over a wide range of $30 \mu$ dust opacities (0.01-2) and dilution factors (0.02-0.5).
\end{abstract}

\section{Introduction}

In this paper we consider the model of radiative pumping through the levels of the second and first torsionally excited states of methanol as described in Sobolev \& Deguchi (1994a) and Sobolev, Cragg \& Godfrey (1997a,b). This model is able to explain the extremely high brightnesses of some MMII as well as ratios of maser line intensities.

In order to figure out at which wavelengths methanol molecules gain and release energy, we explore energy transfer in radiative quantum transitions of methanol under the standard model conditions of Sobolev, Cragg \& Godfrey (1997a). We do this via calculation of net radiative transfer. For a particular quantum transition this quantity corresponds to the difference between the number densities of molecules which increase their energy due to radiative processes and those which undergo energy decrease. The wavelengths of the transitions are well known from methanol spectroscopy. Multiplying the net transfer by the energy difference of the corresponding transition, we obtain the energy acquired by methanol molecules per unit volume from radiation at a particular wavelength. Methanol transitions are very numerous, and the breadths of methanol lines are rather small. At the same time, the variations in dust spectra on a wavelength scale of $0.5 \mu$ are almost negligible. So, in order to make the picture more clear, we summed up the energy acquired by methanol molecules in $0.5 \mu$ wavelength intervals. Results of our calculations for wavelengths in the range up to $200 \mu$ is shown in figure 1 . 


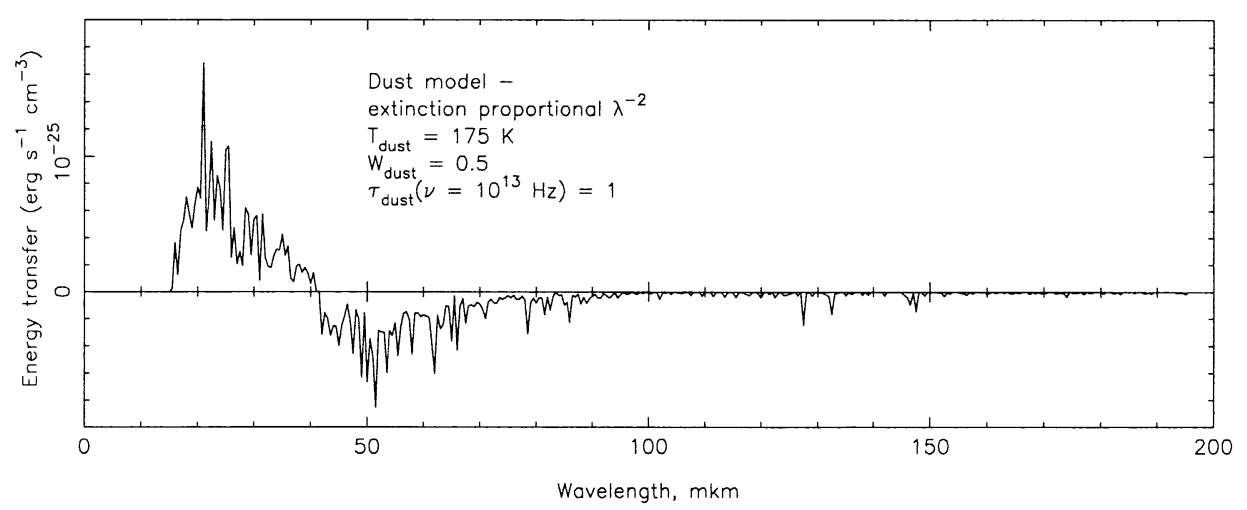

Figure 1. Excitation of methanol molecules due to radiative transitions.

We find that the bulk of the energy transfer for methanol molecules in the masing region fall in the wavelength interval of $15-100 \mu$. It is noteworthy that the contribution of the bright low frequency maser transitions to the overall energy transfer between quantum levels of masing methanol is less then $10^{-6}$. Sobolev \& Deguchi (1994b) have shown that methanol masers are pumped through a very great number of cycles which include transitions between different torsional states. So, in general the methanol maser pumping in the current model is determined by the infrared radiation field.

Figure 1 shows that the masing methanol acquires its energy from radiation field at wavelengths shorter than $41 \mu$ and loses its energy at higher wavelengths. Current models of dust spectra indicate that relation between the energies emitted by dust in the above ranges greatly depends on dust composition, temperature and grain sizes.

\section{Variations in maser spectra due to different characteristics of the pumping dust}

In previous class II methanol maser modeling (Sobolev \& Deguchi 1994a, Sobolev, Cragg \& Godfrey 1997a,b, Zeng \& Lou 1990, and others) a simplified model of the dust radiation was used. Dust opacity was assumed to be proportional to the square of frequency. Such model is computationally simple. The optical properties of this model are close to those of a layer of small silicate particles.

At present, much more refined models of interstellar dust optical properties exist (Draine and Lee 1994, Henning and Stognienko 1996, and others). The dust grains are usually divided into two large groups according to chemical composition of the cores: graphite and silicate grains. Dust models differ in such properties as chemical composition, the presence of mantles, grain size distribution, and grain shape.

For this primary study we have chosen calculations of Draine \& Lee (1994) which reproduce most explicitly basic differences in dust spectra. This paper provides optical constants for chemically homogeneous spheroids consisting of graphite or silicate. The radii of the dust particles vary from $0.001 \mu$ to $10 \mu$. 
In this study we vary the dust parameters of our basic model one at a time. The basic model has the following properties: the optical depth in the middle of the range of effective pumping $(30 \mu)$ is equal to 1 ; the value of the dilution factor for the dust emission is equal to 0.5 ; the dust temperature is equal to $175 \mathrm{~K}$. In order to study regimes when collisional processes are first, negligible and then, influential, we consider hydrogen number densities of $10^{4}$ and $10^{7} \mathrm{~cm}^{-3}$, respectively.
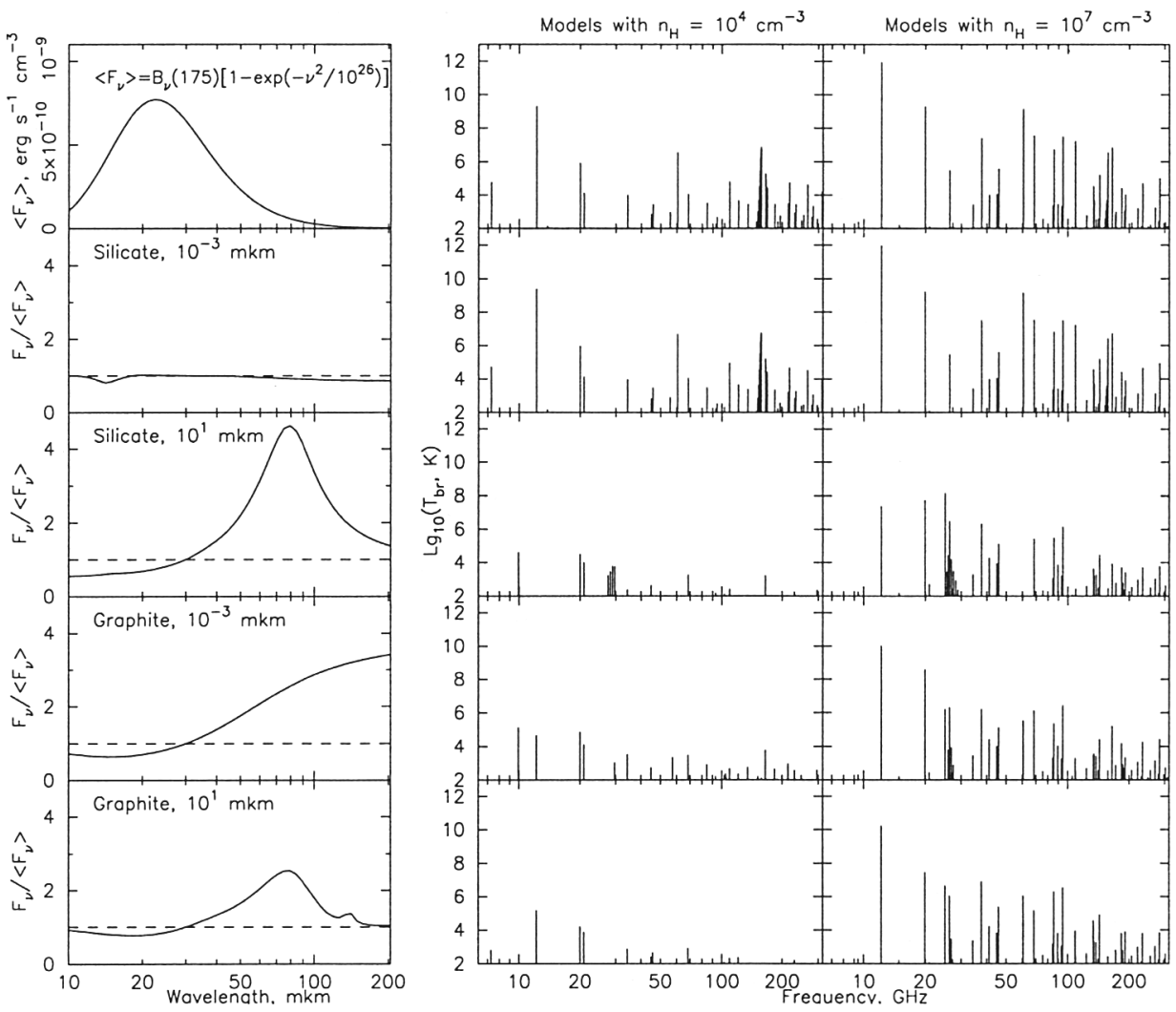

Figure 2. Dust emission models and corresponding maser spectra.

Figure 2 shows the influence of dust chemical composition and grain size on the spectra of class II methanol masers for frequencies $<300 \mathrm{GHz}$. In the left panels the relative intensities of the dust emission with respect to the simple dust model are shown. The right panels show maser spectra for the cases of low and high hydrogen number density. In order to make plots more legible we show E-methanol spectra only.

Our results show that the maser spectra for models with small silicate grains $(0.001-0.01 \mu)$ generally reproduce the observed pattern of maser line ratios (similar to the results obtained with simple dust model). At the same time, models with graphite particles and large silicate particles do not provide satisfactory agreement with the observed ratios of maser lines intensities. Moreover, the brightness temperatures in these models are too small and many observed 
maser lines do not appear. The same results are obtained for a mixture of graphite and silicate dust particles and for silicate dust with a significant fraction of large grains. Thus, interstellar dust in the MMII environment contains mainly silicate grains of small size $(0.001-0.01 \mu)$.

The dependence of maser brightnesses on dust opacity, dust temperature and dilution of dust emission for grains with different chemical composition and grain sizes are studied as well. The flux ratios for six bright maser lines $\left(5_{1}-6_{0} \mathrm{~A}^{+}, 2_{0}-3_{-1} \mathrm{E}, 3_{1}-4_{0} \mathrm{~A}^{+}, 4_{0}-4_{-1} \mathrm{E}, 7_{-2}-8_{-1} \mathrm{E}\right.$, and $\left.7_{2}-6 \mathrm{~A}^{-}\right)$are used to examine the models.

In our calculations the $30 \mu$ dust opacity varies in the range $10^{-3}-2$. In accordance with the results shown in figure 2 , the models with graphite dust of any grain size and silicate dust of large grain size do not reproduce observational data. Some observed lines disappear, and the ratios of brightness temperatures for the bright lines disagree with observed ones. Models with small silicate dust particles do agree with the observational results. The pumping is found to be effective in a wide range of the $30 \mu$ dust opacity from 0.01 to 2 .

The dilution factor for dust emission in our calculations varies from $10^{-3}$ to 0.5. The values of brightness temperatures and the ratios of brightness temperatures for the bright maser lines are close to observed ones only for small silicate grains. The pumping is effective in a wide range of dilution factors from 0.001 to 0.5 . This shows that MMII can be pumped both by relatively distant dusty regions and by the warm dust embedded in maser formation region.

The dependence of maser brightnesses on dust temperature is studied for $0.001 \mu$ silicate grains. The process of pumping is effective when the value of dust temperature is about $125 \mathrm{~K}$ or greater.

\section{Conclusions}

This study shows that the pumping of class II methanol masers by dust emission is effective for dust consisting mainly of small silicate grains. The temperature of the pumping dust should be about $125 \mathrm{~K}$ or greater. The optical depth of the dust at $30 \mu$ can be in the range from 0.01 to 2 . The pumping allows dilution factors of dust emission down to 0.001 .

We thank INTAS (grant 97-11451) and Russian federal program "Astronomy" for financial support.

\section{References}

Draine B.T., Lee H.M. 1984, ApJ, 285, 89

Henning T., Stognienko B. 1996, A\&A, 311, 291

Sobolev A. M., Cragg D. M., Godfrey P. D. 1997a, A\&A, 324, 211

Sobolev A. M., Cragg D. M., Godfrey P. D. 1997b, MNRAS, 288, L39

Sobolev A.M., Deguchi S. 1994a, A\&A, 291, 569

Sobolev A.M., Deguchi S. 1994b, ApJ, 433, 719

Zeng Q., Lou G.F. 1990, A\&A, 228, 480 